\title{
REVIEW
}

\section{MIRROR THERAPY IN ADULT STROKE PATIENTS: A REVIEW OF POSSIBLE APPLICATIONS AND EFFECTIVENESS WITH AN EMPHASIS ON ACTIVITIES OF DAILY LIVING}

\author{
Jan Chrastina $(D$, Hana Svízelová \\ Institute of Special Education Studies, Faculty of Education, Palacký University Olomouc, Olomouc, Czech Republic
}

Received April 6, 2020; Accepted September 16, 2020. Copyright: This is an open access article under the CC BY-NC-ND license.

\begin{abstract}
Aim: To present the current knowledge about possible applications, effectiveness, and other aspects of mirror therapy in adult stroke clients, with an emphasis on the performance of Activities of Daily Living (ADL), or on supporting independence and self-care, including limitations. Design: A descriptive mapping study. Methods: A systematic literature search and an interpretative content analysis (according to predetermined criteria) of 34 identified relevant studies (2000-2017) and conceptualization of findings in relation to ADL performance / limitation among adult stroke clients. Results: Mirror Therapy (MT) is a simple and undemanding approach which improves the function of extremities with respect to performance of ADL. However, due to smaller research samples, it is impossible to clearly confirm its effectiveness and extrapolate the conclusions. MT is also used to complement other therapeutic approaches, or various approaches can be combined with it (e.g., micro-sensory electrical stimulation or observation imitation). Assessment of ADL was primarily performed with the Barthel Index, Modified Barthel Index, and Functional Independence Measures, Functional Ambulation Categories, and Foot and Ankle Ability Measure tests. Conclusion: It seems desirable to apply task-oriented MT - an approach that includes functional tasks to prevent clients from becoming bored during therapy. The performance of MT tasks need not always be supervised by a physiotherapist. Good cooperation between nurses from clinical practice and physiotherapy is desirable. In order to be able to widen the conclusions and clearly confirm the effectiveness of MT in the context of ADL, other randomized controlled (pilot) studies are desirable.
\end{abstract}

Keywords: activities of daily living, adult stroke patients, mirror therapy, stroke, task-oriented mirror therapy.

\section{Introduction}

In the area of various study disciplines and sciences, Mirror Therapy (MT) is a relatively new phenomenon and is still evolving; it may be combined with other approaches, and its applicability extended to cover more diseases and health problems. MT is often recommended to clients with phantom pain after amputation, and to stroke patients. MT can be combined with other therapeutic approaches, or modified as virtual MT. In the application of MT, the principle is visual feedback - a mirror is placed in front of the client, the client's affected extremity is hidden behind it and the mirror reflection of the "healthy" extremity simulates the movement of the affected one (Moseley et al., 2008). This causes mirror neurons to be activated (Najiha et al., 2015) MT was described by V. S. Ramachandran as a way to decrease phantom pain in clients with

Corresponding author: Jan Chrastina, Institute of Special Education Studies, Faculty of Education, Palacký University Olomouc, Žižkovo náměstí 5, Olomouc, Czech Republic; email: jan.chrastina@upol.cz. an amputated extremity (Ramachandran \& RogersRamachandran, 1996). The therapeutic effect is performed using mirrors or boxes (a mirror is on one side and the patient's is extremity hidden inside the mirror box, and, therefore, not visible). MT is a non-pharmacological neuro-rehabilitative approach based on observation of the movements of the intact extremity in the mirror (Bowering et al., 2013; Moseley et al., 2008). The effect of this approach is based on the activation of mirror neurons located in the ventral premotor cortex. These neurons are activated when an individual performs a movement, and during observation of another individual's movements (Najiha et al., 2015).

By placing a mirror in the central sagittal plane, a visual illusion is created and provides positive feedback to the motor cortex. It informs the motor cortex that the patient's affected extremity is moving (Bowering et al., 2013; Najiha et al., 2015). MT is a simple and non-invasive approach, considered a complementary therapy to other procedures (Sütbeyaz et al., 2007). MT was originally developed to relieve phantom pain in post-amputation clients. 
Currently, MT is used in stroke patients, but also in patients suffering from cerebral palsy, Complex Regional Pain Syndrome (CRPS), fractures, and brachial plexus nerve injuries (Grünert-Plüss et al., 2008; Najiha et al., 2015).

\section{Aim}

The aim of the paper is, based on a systematic literature search using a mapping study design, to present the current knowledge about possible applications, effectiveness and other aspects of mirror therapy in adult stroke clients, with an emphasis on the performance of activities of daily living (ADL), or on supporting independence and self-care, including limitations.

\section{Methods}

\section{Design}

A descriptive mapping study was performed, and the authors processed the results and findings based on a mapping study design. This design made it possible to work well with the research problem under investigation in a wide range of available literature and sources, and to suggest further direction for research activities and follow-up studies (Cooper, 2016; Grant \& Booth, 2009). This design did not require flowcharts or flow diagrams, and evidence levels were not considered by the authors. Nevertheless, the authors elaborated a PRISMA flow diagram (Figure 1), used in sorting, classifying, and clustering the investigated studies. During the initial methodological stage, the following working research question was determined for use in the subsequent search activities: "What information about the application of mirror therapy in adult stroke patients in relation to ADL performance / limitations has been published in scientific studies so far?".

\section{Eligibility criteria}

The eligibility criteria were defined as follows: studies focused on the effectiveness of applied MT with a clearly defined target group (adult stroke survivors/adult post-stroke patients), and primarily linked to ADL performance and / or limitations in performance. The required relevant studies were obtained by searching in available electronic databases licensed to Palacký University Olomouc, Czech Republic (EBSCO, PsychINFO, PubMed, and ProQuest), and the meta-search engine, Discovery Service, while full texts were obtained by searching in Google Scholar and ResearchGate.

\section{Search strategy}

The basic search criteria included availability of the full text version of the paper, direct relevance to the research problem, relevance to the research question, and application of MT in adult stroke patients. The authors intentionally searched for studies published in English between 2000 and 2017. In exceptional cases, older resources were used due to their frequent citation, frequent references to them, and relevance / benefits to the area. Papers for which only summaries were available (not full texts) were not included in the search. Empirical studies (without scientific distinction) and review studies (without methodological distinction) were not included in the search process.

Focus on attributes other than the main research topic, focus on different target groups (in terms of age, health status/disease, polymorbidity (where it was not possible to distinguish the health condition and a clear association with stroke), non-survivors, and absence of results concerning ADL or self-care, independence or improvement in motor activities were considered exclusion criteria. The systematic search was based on the following keywords: mirror therap*, mirror box therap*, mirror approach*, stroke*, after stroke patient*, adult*, activit* of daily living, and $A D L$, with Boolean operator combinations. The terms "patient participant - client and therapy - treatment procedure - experiment" were considered to have the same meaning. The authors used an interpretative content analysis to analyze and synthesize their findings. Due to the period of implementation (i.e., 2017), studies from 2018 and later were not searched for or included.

\section{Study selection inc. PRISMA flow diagram}

For sorting, classifying, and clustering the investigated studies, the authors elaborated a PRISMA flow diagram (Figure 1). Of the 1,323 primarily searched studies, 89 were subordinated to the screening phase. A total of 45 studies met the eligibility criteria, and 34 were included in the secondary (final) sample. Eleven full-text studies were excluded because they focused on more thematic areas (with minimal attention devoted to MT), and because the impact of MT on ADL was not unequivocally proven in the study.

\section{Evaluation of quality of articles}

Quality assessments were performed separately by both authors, using a data extraction sheet of their own design (see data extraction section below). During the evaluation of the quality of studies, agreement between the two authors was achieved independently. Since the paper was not designed as a systematic / literature review, evidence levels were not referred to. 


\section{Data extraction}

The authors performed data extraction using a data extraction sheet of their own design. It contained columns including authors, study titles, research designs, aims / objectives, recommendations / conclusion, and main outcomes. In the first phase, this source document sheet was not shared between the two authors. After an autonomous assessment of the studies by both authors, a final version of the data extraction sheet containing their matches was created. The summary of documents included in the subsequent analysis and processing included the selection (secondary) sample of documents. These were documents meeting the search criteria and acceptance criteria. Any duplicates of full texts were rejected. During analysis of the studies in the selection sample, studies were rejected that did not focus on the issue in question or included only references to MT and the results of its application. After analytical and systematic processing of the content of the identified studies, 34 relevant studies published in English were used to produce the mapping study.

\section{Limitation of study}

The processing of this study was part of the broader scientific and research activities of the team of authors. Therefore, the authors did not present the overall (primary) set of identified studies / documents (regarding the various objectives of the overall research aims) but only their systematic work on the selection (secondary) set, consisting of studies that used MT in adult stroke patients (see above) - one of the partial objectives defined by the authors of this study. The authors accept that possibly not all published studies have been identified, and that relevant studies published in languages other than English have not been included in the review.

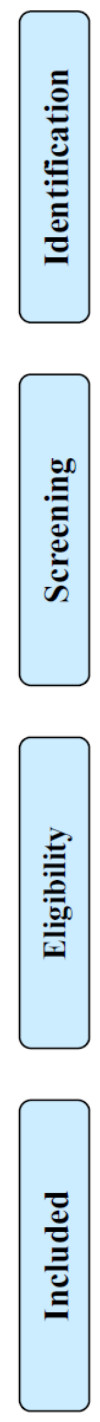

Records identified through database searching $(\mathrm{n}=1,323)$
Additional records identified through other sources $(\mathrm{n}=0)$

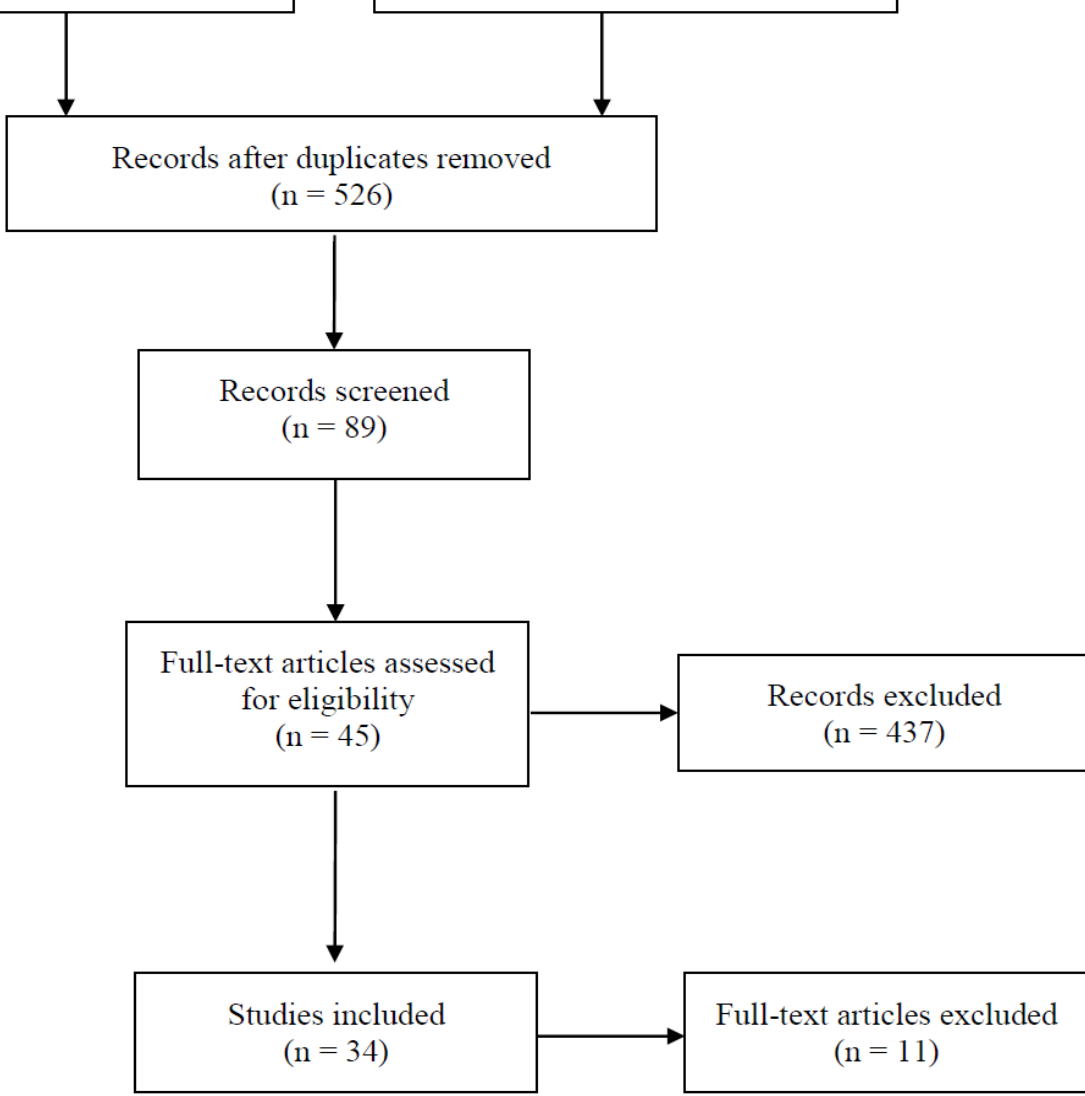

Figure 1 PRISMA 


\section{Results}

Mirror therapy in adult stroke patients and its effects on the performance of $A D L$

The studies identified by the search describe and distinguish the application of MT to ameliorate the effects of stroke on the function of upper and lower extremities and on activities of daily living (taking into account the various stages of stroke). Paresis of the upper extremity is a common consequence of stroke. As many as $85 \%$ of stroke survivors are affected by hemiparesis, and 55 to $75 \%$ of patients suffer permanently limited function of the upper extremity. MT is an attractive treatment alternative due to its simplicity, cost-effectiveness, and absence of concerns on the part of patients (Yavuzer et al., 2008). During MT, a mirror is placed between the arms (upper extremities) or legs (lower extremities) of the patient so that the reflection of the healthy extremity creates the illusion of normal movement of the affected extremity (Thieme et al., 2012). Paresis of the upper or lower extremity, a frequent consequence of stroke, causes problems in the performance of ADL, such as walking, dressing, or eating (Thieme et al., 2012). Most stroke patients are affected by motor impairment of the upper extremity, resulting in ADL limitations (Lee et al., 2015); ADL are largely limited by unsuccessful recovery of distal joints of the wrist and fingers, despite the restoration of the required strength and coordination of proximal joints (arm and elbow) (Rajappan et al., 2015).

Two thirds of patients with lower extremity impairment are unable to walk independently immediately after stroke, and only a half of patients are capable of independent walking after rehabilitation (Michielsen et al., 2011). During the first 12 months after stroke, almost $50 \%$ of patients feel pain in the upper extremity, especially in the shoulder region, and suffer from the presence of type 1 CRPS (Jönsson et al., 2006), and this pain decreases quality of life (Lindgren et al., 2007; Thieme et al., 2012). The improvement of patient self-care is one of the most important aspects of ADL performance (Yavuzer et al., 2008). If patients are unable to perform these activities autonomously, independent life is impossible and they must rely on the family or other sources of support (Park et al., 2015).

Many studies have shown that MT can significantly improve motor functions of the upper extremity and ADL performance in stroke patients. A study was carried out in which MT combined with micro-sensory electrical stimulation (group MT + SES) was compared with MT only (group MT) and MT with sham micro-sensory electrical stimulation (group MT
+ sham SES). During rehabilitation in all groups, ADL were performed (for example picking up a plastic bag with the affected hand, squeezing a towel with both hands, and stabilizing a glass of water with one hand while taking food with the other). These ADL were then assessed by means of the Functional Independence Measures (FIM) test (consisting of 13 motor and five cognitive tasks) using a seven-point scale. The study concluded that the score of motor tasks in the FIM test significantly differed between the group of MT + sham SES, and the other two groups. A subsequent analysis revealed that the group of MT + SES and MT + sham SES showed a higher degree of functional independence compared with the MT only group (Lee et al., 2015).

A similar study was conducted by Lin et al. (2014a) but instead of MT + sham SES, the authors used a control group with no form of MT. In terms of ADL, there were no differences between the groups after four weeks of therapy. In a previous study by Lin et al. (2014b), MT was combined with sensory electrical stimulation to identify a possible effect on ADL. A total of 16 patients were randomly divided into two groups: MT combined with sensory electrical stimulation (MT + SES) and MT only. The participants were asked to carry out symmetrical movements with both arms as simultaneously as possible, while in the mirror they watched the reflection of the healthy arm as if it were the affected arm. The activities included movements with objects (building with blocks or turning cards) and movements without objects (pronation and supination of the forearm or touching fingers). In the MT + SES group, the participants had a stimulation glove on the affected hand in the course of the therapy. The performance of ADL was assessed using the FIM test. The final score did not indicate any significant differences between the groups. However, an improvement was observed in the "Transfers" subarea in the MT + SES group. No significant differences were observed in the overall score or other sub-areas (Lin et al., 2014b).

Park et al. (2015) also investigated MT of the upper extremity and its effect on ADL in hemiplegic stroke patients, conducting a study involving 30 chronic stroke patients, who had suffered from hemiplegia for at least six months. The patients were divided into a "mirror group" and a "control group" and their ability to carry out ADL was assessed. The resulting comparison of the participants' abilities to perform ADL showed statistically significant differences between the two groups, especially in terms of selfcare. In Sphincter control, Transfers, Locomotion, Communication, and Social cognition, no significant differences were observed between the groups. This 
study suggested a positive effect of MT in the area of ADL in chronic stroke patients.

Thieme et al. (2013) carried out a study involving three groups of subacute stroke patients with severe paresis of the upper extremity. The first group underwent individualized MT, the second group underwent group MT, and the third group was a control group. One of the main criteria was independence in the performance of ADL, using the Barthel Index (BI). The study concluded that MT was no more effective in terms of independent performance of ADL compared with the control group. The main limitation of the study was the severe impairment of subacute stroke patients, and, therefore, the results could not be generalized to include other stroke patients.

Probably the first study aimed at MT of the lower extremities and the effect on ADL was published by Sütbeyaz et al. (2007) involving 40 patients who had suffered a stroke during the previous 12 months. The participants were divided into an MT group and a control group. Both groups underwent standard therapy. In addition, the MT group underwent 30 minutes of MT a day aimed at a lower extremity, performed in a semi-sitting position in bed. The participants watched the movement of the unaffected extremity in a mirror. The control group underwent the same procedure, but the reverse (nonreflective) side of the mirror was used. The ability to walk was assessed with the Functional Ambulation Categories (FAC) test; and motor skills assessed with the FIM test. The participants were allowed to use walking / locomotion aids (for example a walking stick). According to the results, MT combined with conventional stroke treatment brings long-lasting motor function improvement in subacute stroke patients.

The effectiveness of MT aimed at the lower extremity in hemiparetic stroke patients in the context of ADL was also investigated by Cortez et al. (2016). In their study, a total of 20 participants underwent MT in combination with conventional physiotherapy. All participants undertook identical tasks and were not divided into groups. The results were assessed by means of the Foot and Ankle Ability Measure test, which comprises two subscales - ADL (21 items), and a sports subscale (eight items). In this experimental study, only the ADL subscale was considered. The results indicated a significant improvement after ADL-related intervention.

Mirror therapy of the upper extremity in adult patients with subacute and chronic stroke

Most of the identified studies on MT of the upper extremity in stroke patients focused in particular on subacute and chronic stroke patients (Colomer et al., 2016; Invernizzi et al., 2013; Lee et al., 2015; Lim et al., 2016; Lin et al., 2014a; 2014b; Paik et al., 2014; Park et al., 2015; Rajappan et al., 2015; Rodrigues et al., 2016; Sciusco et al., 2008; Selles et al., 2014; Thieme et al., 2013).

Sciusco et al. (2008) performed a study involving 14 subacute or chronic stroke patients (an MT group and control group underwent conventional therapy), in which MT was combined with observation-based imitation. The participants watched an actor's movements and then tried to imitate the movements as closely as possible. This approach is recommended when used in addition to conventional therapy due to its effectiveness, simplicity and feasibility. MT in combination with observation-based imitation could become an effective approach to ensure better recovery of the upper extremity in subacute or chronic stroke patients.

Similarly, Invernizzi et al. (2013) performed a study aimed at MT of the upper extremity in subacute stroke patients. A sample of 24 patients was divided into an MT group and a control group. Both groups underwent conventional therapy; the MT group had an additional 30 minutes of MT and one hour of MT a day for the remaining two weeks. The control group underwent the same program but with a hidden mirror (sham MT). Eventually, it was confirmed that MT used in conjunction with conventional therapy was more beneficial in terms of restoration of motor functions of the upper extremity compared with conventional therapy alone.

A similar study was conducted by Colomer et al. (2016). However, their participants were chronic stroke patients and the control group underwent only passive mobilization of the affected extremity. In both groups, improvement in motor functions was observed. Nevertheless, the group that underwent MT showed a significant improvement in tactile perception, which was observed primarily in the form of increased sensitivity to fine touches.

The effect of MT on subacute stroke patients with upper extremity hemiparesis was also examined by Rajappan et al. (2015). A total of 30 participants who had suffered a stroke two to 12 months before the study were randomly divided into a group undergoing MT and a group undergoing sham MT.

During the therapy, the patients sat near a table with a vertically positioned mirror in the sagittal plane. The paretic arm was placed behind the mirror and the non-paretic arm in front of the mirror. As a result, the patients only saw the unaffected extremity. They performed simple movements with the unaffected extremity (such as bending and stretching the fingers, 
bending and stretching the wrist, ulnar and radial deviation) and specific tasks (such as grasping and squeezing objects of various sizes and weights). During the movements performed by the unaffected extremity, the participants concentrated all their attention on the mirror and watched the reflection of the unaffected extremity, with the aim of performing identical movements with the affected extremity. In the group that underwent sham MT, the same tasks were performed but the non-reflective side of the mirror was used. The results showed a significant improvement in both groups in motor and functional areas (the MT group achieved more significant improvement compared with the sham MT group in both monitored areas - motor and functional).

Selles et al. (2014) performed a research study that surpassed all others. The study involved a total of 93 chronic stroke patients, representing a large test group compared with other studies. The execution of the study was also unconventional - the patients were divided into five groups: Group 1) exercised the paretic arm observed directly (paretic group without mirror); Group 2) exercised the non-paretic arm observed directly (non-paretic group without mirror); Group 3) exercised the non-paretic arm reflected in the mirror (non-paretic group with mirror); Group 4) exercised both extremities with a curtain to prevent the view of the paretic extremity (double-sided group with curtain); and Group 5) exercised both extremities with a reflection of the non-paretic arm in the mirror (double-sided group with mirror).

The results of the study suggest that the use of mirror reflection can facilitate motor learning, as confirmed by relatively high effectiveness in performing the exercise/movement with the unaffected extremity only. The data also indicate that MT is no more effective than direct exercising of the affected extremity. The authors conclude that MT can be effective in specific situations (e.g. when the patient is still unable to move the affected extremity, or the movement is very tiring).

Mirror therapy of the upper extremity in adult patients with acute stroke

While most authors focused mainly on the effectiveness of MT of the upper extremity, especially on motor functions in subacute or chronic stroke patients, Yeldan et al. (2015) conducted a study involving stroke patients at a very early acute stage. They investigated the effect of early MT on functional improvement of the upper extremity, whereby the therapy is applied to hospitalized patients immediately after stroke. A total of 120 patients were assessed in terms of suitability for this study; the final sample included 30 persons (who had suffered a stroke less than one month before the study), but only eight completed the programme successfully. The participants were divided into two groups (the first group underwent MT + neuro-developmental therapy, and the second underwent neurodevelopmental therapy only). To determine the degree of independence in functional activities, the ten-item BI was used. The patients in the group of neurodevelopmental therapy underwent an individualized therapeutic session based on the Bobath concept, lasting 40 minutes. In the MT group, each participant underwent (in addition to neuro-developmental therapy) a very early 20-minute MT program. The programs started when the participants were hospitalized and continued on an outpatient basis after discharge from hospital. The patients were instructed to focus solely on the reflection in the mirror (Yeldan et al., 2015). The very early MT program was designed to improve motor functions of the upper extremity involving specific movements used in ADL. The taskoriented activities in this program were individually designed for each patient. The complexity of the tasks increased according to the patient's ability to concentrate. The interventions were carried out by the participants individually under physiotherapist supervision. The results suggested improvement in BI in both groups (two patients from each group). This study showed that very early MT did not have an additional effect on improved function of the upper extremity in acute stroke patients.

According to a different research study conducted by Radajewska et al. (2013), MT improves the ability of independent performance of ADL in stroke patients with right-sided paresis of the upper extremity. The study included 60 right-handed patients who suffered ischaemic stroke eight to ten weeks before the study. They were randomly divided into two groups an MT group and control group. In each group, two subgroups were created. One of the subgroups included patients with right-sided paresis, while the other group included patients with left-sided paresis (each subgroup had 15 patients). The MT group underwent additional exercise with a mirror. The control group underwent conventional treatment without MT. To assess self-care in the area of daily activities, the Functional Index "Repty" (FIR) scale was applied. The results suggested a significant improvement in this area in the right-sided paresis subgroup of the group undergoing MT.

\section{Mirror therapy of the lower extremity in adult stroke patients}

To present evidence about the effectiveness of MT of the lower extremity is very problematic due to the low number and diversity of relevant studies. 
Available studies vary in treatment specifications at various stages after stroke (Hung et al., 2015). A number of studies (Cortez et al., 2016; Sütbeyaz et al., 2007) focused on MT of the lower extremity and the effect on ADL.

Ji and Kim (2015) dealt with MT of the lower extremity in subacute stroke patients using a control group undergoing sham MT. According to the results, MT in addition to conventional stroke treatment might be beneficial for posture improvement in subacute stroke patients.

Mohan et al. (2013) and Wada et al. (2011) analyzed MT of the lower extremity in acute stroke patients, while Salem and Huang (2015) focused on chronic stroke patients. Mohan et al. (2013) conducted a study involving 22 patients divided into an MT group and a control group. Both groups underwent conventional therapy. In addition, the MT group underwent 30 minutes of MT, including functional movements using unaffected joints of the hip, knee and ankle performed in a sitting or semi-sitting position. In the MT group, the reflective surface of the mirror was turned to reflect the non-paretic extremity. In the control group, the non-reflective side of the mirror was turned to the non-paretic extremity. However, the authors concluded that the two-week MT of the lower extremity in acute stroke patients combined with conventional therapy (in comparison with conventional therapy without MT) did not bring any substantial improvement.

Wada et al. (2011) performed a study aimed at acute stroke patients but involving only nine participants without a control group (with particular focus on ankle function). No significant differences were observed between the results of individual participants. However, there were differences before and after therapy.

Salem and Huang (2015), who focused on MT of the lower extremity in chronic stroke patients, performed a study involving 30 patients divided into two groups: an MT group and a control group. Both groups underwent conventional therapy. The authors concluded that MT combined with conventional therapy may improve motor functions of the lower extremity and walking speed in chronic stroke patients.

\section{Task-oriented mirror therapy in adult stroke patients}

Paik et al. (2014) emphasize that not all studies addressing MT in stroke patients bring promising results. Baek (2009) observed that the effect of MT decreased with time, but also that although MT involving repetition of simple movements brought improvement during the first four weeks of treatment, it was followed by a gradual loss of functions once the patients became bored. This resulted in a certain therapy resistance. According to the author, the therapy seems to be more efficient if MT includes elements of functional tasks than if it contains only simple imitation movements.

Yoo (2010) also suggested the use of multiple functional and task-oriented programs because simple movements limited functional recovery. Although MT showed improvement in motor functions in stroke patients, the differences between various concepts and tasks caused inconsistencies in their results and conclusions.

Previous studies on the use of MT always compared the results with a control group who had not undergone MT (Baek, 2009). Therefore, Paik et al. (2014) decided to carry out a study aimed at a comparison of the effectiveness of MT involving simple and taskoriented movements during functional recovery of the upper extremity in patients with hemiplegia after stroke (all participants had suffered stroke 17 months or more before the study). Simple MT included, for example, pronation and supination of the forearm, stretching and bending the fingers or "counting the fingers". During the task-oriented part of the therapy the participants performed movements associated with ADL - grasping and releasing balloons, pressing pliers, using a spray, kneading, grasping a coin, using a spoon, lifting a heavy can, and wiping a table. In all patients, the function of the upper extremity was assessed by means of the Box and Blocks Test (BBT), Cube Carry, and Card Turning Tests. Similarly, in the course of this study, the patients with simple MT grew bored and resistant to the therapy. On the other hand, patients undergoing task-oriented MT maintained their concentration and interest in the movements. This study concluded that the function of the upper extremity improved more after task-oriented therapy compared with simple MT.

Based on previous studies, Rodrigues et al. (2016) decided to refine MT with various objects that would improve the level of activity during the performance of single-sided and double-sided activities (adding object-related bilateral symmetrical training). The author considered the use of objects in MT a challenge, because the aim of this therapy is to create an illusion of normal movements of the paretic arm and therefore, the illusion should simulate symmetrical movement of both extremities. For the purposes of creating this illusion, the object may be halved, and each half placed on either side of the mirror. Both groups underwent one hour of objectrelated bilateral symmetrical training. While the MT group performed bilateral tasks using a mirror, the control group had the mirror covered. In the execution 
of the tasks, the participants used daily objects such as a bottle or a ball. The participants in the MT group were instructed to perform simultaneous movements of the hand and arm, and to concentrate on the reflection of the unaffected extremity in the mirror. The control group performed the same movement, but the mirror was covered with paper, so they observed the movement of the affected extremity. The exercise was performed in the patients' home environment to enable full concentration on the activities. During the training procedure, both groups were supervised by a physiotherapist. Overall, nine of the 15 components of task-oriented training activities were followed-up. The results showed improvement in the performance and quality of the movements in the control group and suggested that bilateral training with concentration on the paretic upper extremity brought better results compared with the same training using a mirror (Rodrigues et al., 2016).

Lim et al. (2016) carried out a study with 60 patients. The aim was to demonstrate the effectiveness of taskoriented MT in subacute stroke patients using an analysis of improved function of the upper extremity and in ADL in patients who had undergone MT compared with those who had been subject to sham therapy. The patients were divided into two groups. The MT group patients underwent MT by imitating the mirror reflection of the unaffected upper extremity. The patients tried to move the hemiplegic extremity in the same way as the unaffected extremity reflected in the mirror. This was performed under a therapist's supervision, who helped the patients concentrate on the movements. The functional tasks in the first week were simple movements (pronation and supination of the forearm, bending and stretching of the wrist). In the second week, the activities included bending and stretching of the patients' fingers, "counting the fingers", and tapping and touching the fingers. In the third week, the patients performed simple manipulation tasks (such as picking up coins and beans, turning cards, and placing blocks in a container). During the fourth week, the patients performed more complex tasks, such as attaching pins to a perforated board and removing them, drawing simple pictures and colouring. The sham therapy group performed the same tasks at the same time. However, between the hemiplegic and unaffected extremity a wooden board was installed instead of a mirror. ADL were then assessed by means of the Modified Barthel Index. As a result, the MT group saw the reflection of the unaffected extremity, but in the sham therapy group the wooden board prevented view of the unaffected extremity.

The authors concluded that MT involving functional tasks had significant effects in terms of improvement of the functions of the upper extremity and the ability to carry out ADL in subacute stroke patients. On the basis of these findings, MT with functional tasks may serve as an alternative form of rehabilitation for stroke patients.

\section{Discussion}

A frequently investigated issue in this area was the use of MT in stroke patients and its effect on ADL (Cortez et al., 2016; Lee et al., 2015; Lin et al., 2014a; Lin et al., 2014b; Park et al., 2015; Sütbeyaz et al., 2007; Thieme et al., 2013). Most authors examined the phenomenon of ADL in chronic stroke clients (Cortez et al., 2016; Lee et al., 2015; Lin et al., 2014a; Lin et al., 2014b; Park et al., 2015). Sütbeyaz et al. (2007) and Thieme et al. (2013) focused on subacute stroke patients. A majority of studies addressed MT of the upper extremity and its effect on ADL (Lee et al., 2015; Lin et al., 2014a; Lin et al., 2014b; Park et al., 2015; Thieme et al., 2013), only Cortez et al. (2016) and Sütbeyaz et al. (2007) focused on the lower extremity. The most frequent combination of MT and other treatment approach focusing on ADL was microsensory electrical stimulation (Lee et al., 2015; Lin et al., 2014a; Lin et al., 2014b). Most studies used a control group for comparison reasons (Lin et al., 2014a; Lin et al., 2014b; Park et al., 2015; Sütbeyaz et al., 2007; Thieme et al., 2013). In terms of results, almost all studies reported a degree of improvement in extremity function and effect on ADL (Cortez et al., 2016; Lee et al., 2015; Lin et al., 2014a; Lin et al., 2014b; Park et al., 2015; Sütbeyaz et al., 2007). Other authors who did not analyze MT in the context of ADL mostly focused on MT of the upper extremity in subacute or chronic stroke patients (Colomer et al., 2016; Invernizzi et al., 2013; Rajappan et al., 2015; Sciusco et al., 2008; Selles et al., 2014). Regarding relevant studies, MT of the upper extremity in acute patients was analyzed by Radajewska et al. (2013) and Yeldan et al. (2015). All studies focusing on MT of the upper extremity in subacute or chronic adult stroke patients reported more or less positive results, while those studies that involved acute stroke patients reported equivocal results. Another area of research was task-oriented MT - including functional tasks in order to prevent the participants from becoming bored, which might result in a loss of concentration on movements. As this is a relatively new phenomenon, mentioned for the first time in a study by Baek (2009), only three published studies were identified focusing on this area (Lim et al., 2016; Paik et al., 2014; Rodrigues et al., 2016). The study mentioned above deals with the effect of task-oriented MT on ADL, with seemingly very promising results. All these studies focused solely on MT of the upper extremity 
in subacute (Lim et al., 2016) or chronic (Paik et al., 2014; Rodrigues et al., 2016) adult stroke patients.

In the area of nursing care and research, the effect of the connection between MT and acupuncture (Zhang et al., 2018a) and "Tong Du Xing Shen" acupuncture was monitored (functional recovery of posterior lower extremity in post-stroke patients) (Zhang et al., 2018b). Activities of the upper limbs were assessed using the Fugl-Meyer Motor Function test (FMA), Brunnstrom upper limb and hand staging (The Brunnstrom Approach), the BI test, and hemiplegia dorsal wrist active range of joint activities (AROM). The results of the study show that MT combined with acupuncture helps to improve patients' upper limb motor functions, which contributes to an increase in ADL level. Nursing interventions based on the results of MT and traditional acupuncture can improve the motor functioning in adult stroke patients (lower extremity dysfunction).

In rehabilitative nursing, a practical nursing plan designed for patients with severed finger reunion can also be used (Lan et al., 2016). In the area of stroke rehabilitation, an interesting option, exemplified by a randomized trial, is the combination of functional electrical stimulation (FES) and MT, applied in poststroke patients in the context of upper limb motor recovery. To assess the effectiveness of this combination, the authors used the Action Research Arm Test and the Fugl-Meyer Upper Extremity assessment. Good cooperation between nurses from clinical practice and physiotherapy is desirable (Mathieson et al., 2018).

The findings of a systematic review and meta-analysis by Louie et al. (2019) are also important for clinical nursing practice. The authors demonstrated that MT applied to the lower extremity of stroke patients produced a valuable improvement in gait speed. The authors also demonstrated a small improvement in the areas of mobility and lower extremity motor skills recovery. The authors see its greatest importance being in use with subacute stroke patients.

Evidence relevant to clinical nursing practice and its research is supported by the conclusions of a study by Yildirim and Kanan (2016), who state that MT can be performed by patients independently - helping to increase patients' self-control and self-management. It should be included in the nursing care plan for its safety and feasibility.

\section{Conclusion}

MT is a safe therapy that can be performed in a sitting position, in both hospitalized and home care clients (Louie et al., 2019). The performance of MT tasks need not always be supervised by a physiotherapist. Although MT is a relatively new phenomenon, this approach is well-known internationally and the scope of application of this highly specific therapy continues to increase. In the Czech Republic (the country of the authors of the present study), this issue is not well elaborated, and MT is used sporadically.

MT is still considered an experimental type of treatment in clients for whom physiotherapeutic, pharmaco-therapeutic, and other approaches have failed. Due to the fact that the therapy is frequently modified and combined with other approaches, and on various samples of patients (and various diseases), its effectiveness cannot be fully generalized.

The studies identified for the purposes of this review were primarily randomized controlled (pilot) studies involving a control group. Some addressed MT and its effect on ADL with almost all emphasizing a degree of improvement in extremity function and ADL. Attention was also given to MT of the upper extremity in subacute or chronic stroke patients, with predominantly positive results. An insufficiently researched and published aspect is task-oriented MT. However, so far, little is known about the results of long-term follow-up in post-stroke patients suffering from after-stroke hemiparesis, and there is no clearly defined time when MT should be applied (Toh \& Fong, 2012). It seems appropriate to use MP in holistically-oriented nursing care, which would combine nurse interventions with those of specialists in the fields of psychology, occupational therapy, social work and others. Education in MT training is simple and time-saving and should become a component in professional training in nursing and therapeutic interventions. Further research activities could be focused on the experience of post-stroke patients with MT therapy themselves. MT remains an appropriate and indispensable topic for nursing management.

\section{Ethical aspects and conflict of interest}

The authors state that they are not aware of any conflict of interest regarding this paper. All authors have read and approved the submitted manuscript and it has not been submitted or published elsewhere, in whole or in part, except as an abstract. The authors also declare that the text includes appropriate citations of all bibliographical sources.

\section{Funding}

This paper was supported by the following project: End of life and dying in persons with disabilities: a meta-synthesis (GF_PdF_2019_0007), Faculty of Education Dean's Grant. 


\section{Author contributions}

Conception and design ( $\mathrm{JCH}, \mathrm{HS})$, data collection (HS), data analysis and interpretation (JCH, HS), manuscript draft $(\mathrm{JCH})$, critical revision of the manuscript $(\mathrm{JCH})$, final approval of the manuscript (JCH, HS).

\section{References}

Baek, N. Y. (2009). Effects of mirror therapy on hemiplegia in the function of lower extremity [Unpublished master's thesis]. Department of Physical Therapy, Graduate School of Rehabilitation Science, Daegu University.

Bowering, K. J., O’Connell, N. E., Tabor, A., Catley, M. J., Leake, H. B., Moseley, G. L., \& Stanton, T. R. (2013). The effects of graded motor imagery and its components on chronic pain: a systematic review and meta-analysis. The Journal of Pain, 14(1), 3-13. https://doi.org/10.1016/i.jpain.2012.09.007

Colomer, C., Noé, E., \& Llorens, R. (2016). Mirror therapy in chronic stroke survivors with severely impaired upper limb function: a randomized controlled trial. European Journal of Physical and Rehabilitation Medicine, 52(3), 271-278.

Cooper, D. (2016). What is a "mapping study?" Journal of the Medical Library Association, 104(1), 76-78.

https://dx.doi.org/10.3163\%2F1536-5050.104.1.013

Cortez, B. V., Coêlho, C. K. S., Silva, D. R. C., Oliveira, M. C. B., Oliveira, G. B. V. P., Sousa e Silva, F. M., Frederico, É. H. F. F., Sá-Caputo, D. C., Bernardo-Filho, M., \& Silva, J. M. (2016). Effects of mirror therapy on the lower limb functionality hemiparesis after stroke. Health, 8(14), 14421452. http://dx.doi.org/10.4236/health.2016.814144

Grant, M. J., \& Booth, A. (2009). A typology of reviews: an analysis of 14 review types and associated methodologies. Health Information and Libraries Journal, 26(2), 91-108. https://doi.org/10.1111/j.1471-1842.2009.00848.x

Grünert-Plüss, N., Hufschmid, U., Santschi, L., \& Grünert, J. (2008). Mirror therapy in hand rehabilitation: a review of the literature, the St Gallen Protocol for mirror therapy and evaluation of a case series of 52 patients. The British Journal of Hand Therapy, 13(1), 4-11. https://doi.org/10.1177\%2F175899830801300101

Hung, G. K. N., Li, C. T. L., Yiu, A. M., \& Fong, K. N. K. (2015). Systematic review: effectiveness of mirror therapy for lower extremity post-stroke. Hong Kong Journal of Occupational Therapy, 26(1), 51-59.

https://doi.org/10.1016/j.hkjot.2015.12.003

Invernizzi, M., Negrini, S., Carda, S., Lanzotti, L., Cisari, C., $\&$ Baricich, A. (2013). The value of adding mirror therapy for upper limb motor recovery of subacute stroke patients: a randomized controlled trial. European Journal of Physical and Rehabilitation Medicine, 49(3), 311-317.

Ji, S. G., \& Kim, M. K. (2015). The effects of mirror therapy on the gait of subacute stroke patients: a randomized controlled trial. Clinical Rehabilitation, 29(4), 348-354. https://doi.org/10.1177/0269215514542356

Jönsson, A. C., Lindgren, I., Hallström, B., Norrving, B., \& Lindgren, A. (2006). Prevalence and intensity of pain after stroke: a population based study focusing on patients' perspectives. Journal of Neurology, Neurosurgery, and Psychiatry, 77(5), 590-595.

https://dx.doi.org/10.1136\%2Fjnnp.2005.079145
Lan, M., Huiling, L., Yuhong, F., Jun, W., Jun, Y., Weixiang, Y., \& Liping, Z. (2016). Study on construction scheme of mirror therapy rehabilitation nursing in patients with severed finger reunion on the basis of Delphi method. Chinese Journal of Modern Nursing, 22(29), 4193-4196. http://dx.chinadoi.cn/10.3760/cma.j.issn.16742907.2016.29.012

Lee, Y. Y., Lin, K. C., Wu, C. Y., Liao, C. H., Lin, J. C., \& Chen, C. L. (2015). Combining afferent stimulation and mirror therapy for improving muscular, sensorimotor, and daily functions after chronic stroke: a randomized, placebocontrolled study. American Journal of Physical Medicine \& Rehabilitation, 94(10), 859-868. https://doi.org/10.1097/phm.0000000000000271

Lim, K. B., Lee, H. J., Yoo, J., Yun, H. J., \& Hwang, H. J. (2016). Efficacy of Mirror Therapy Containing Functional Tasks in Poststroke Patients. Annals of Rehabilitation Medicine, 40(4), 629-636.

https://dx.doi.org/10.5535\%2Farm.2016.40.4.629

Lin, K. C., Huang, P. C., Chen, Y. T., Wu, C. Y., \& Huang, W. L. (2014a). Combining afferent stimulation and mirror therapy for rehabilitating motor function, motor control, ambulation, and daily functions after stroke. Neurorehabilitation and Neural Repair, 28(2), 153-162. https://doi.org/10.1177/1545968313508468

Lin, K. C., Chen, Y. T., Huang, P. C., Wu, C. Y., Huang, W. L., Yang, H. W., Lai, H. T., \& Lu, H. J. (2014b). Effect of mirror therapy combined with somatosensory stimulation on motor recovery and daily function in stroke patients: a pilot study. Journal of the Formosan Medical Association, 113(7), 422-428. https://doi.org/10.1016/j.jfma.2012.08.008

Lindgren, I., Jönsson, A. C., Norrving, B., \& Lindgren, A. (2007). Shoulder pain after stroke: a prospective populationbased study. Stroke, 38(2), 343-348. https://doi.org/10.1161/01.str.0000254598.16739.4e

Louie, D. R., Lim, S. B., \& Eng, J. J. (2019). The Efficacy of Lower Extremity Mirror Therapy for Improving Balance, Gait, and Motor Function Poststroke: A Systematic Review and Meta-Analysis. Journal of Stroke and Cerebrovascular Diseases, 28(1), 107-120.

https://doi.org/10.1016/j.jstrokecerebrovasdis.2018.09.017

Mathieson, S., Parsons, J., Kaplan, M., \& Parsons, M. (2018). Combining functional electrical stimulation and mirror therapy for upper limb motor recovery following stroke: a randomised trial. European Journal of Physiotherapy, 20(4), 244-249. https://doi.org/10.1080/21679169.2018.1472635

Michielsen, M. E., Selles, R. W., van der Geest, J. N., Eckhardt, M., Yavuzer, G., Stam, H. J., Smits, M., Ribbers, G. M., \& Bussmann, J. B. (2011). Motor recovery and cortical reorganization after mirror therapy in chronic stroke patients: a phase II randomized controlled trial. Neurorehabilitation and Neural Repair, 25(3), 223-233. https://doi.org/10.1177/1545968310385127

Mohan, U., Babu, S. K., Kumar, K. V., Suresh, B. V., Misri, Z. K., \& Chakrapani, M. (2013). Effectiveness of mirror therapy on lower extremity motor recovery, balance and mobility in patients with acute stroke: a randomized sham-controlled pilot trial. Annals of Indian Academy of Neurology, 16(4), 634-639. https://doi.org/10.4103/0972-2327.120496

Moseley, G. L., Gallace, A., \& Spence, C. (2008). Is mirror therapy all it is cracked up to be? Current evidence and future directions. Pain, 138(1), 7-10.

https://doi.org/10.1016/j.pain.2008.06.026 
Najiha, A., Alagesan, J., Rathod, V. J., \& Paranthaman, P. (2015). Mirror therapy: a review of evidences. International Journal of Physiotherapy and Research, 3(3), 1086-1090. http://dx.doi.org/10.16965/ijpr.2015.148

Paik, Y-R., Kim, S-K., Lee, J-S., \& Jeon, B-J. (2014). Simple and task-oriented mirror therapy for upper extremity function in stroke patients: a pilot study. Hong Kong Journal of Occupational Therapy, 24(1), 6-12. https://doi.org/10.1016\%2Fj.hkjot.2014.01.002

Park, J. Y., Chang, M., Kim, K. M., \& Kim, H. J. (2015). The effect of mirror therapy on upper-extremity function and activities of daily living in stroke patients. Journal of Physical Therapy Science, 27(6), 1681-1683. https://dx.doi.org/10.1589\%2Fjpts.27.1681

Radajewska, A., Opara, J. A., Kucio, C., Błaszczyszyn, M., Mehlich, K., \& Szczygiel, J. (2013). The effects of mirror therapy on arm and hand function in subacute stroke in patients. International Journal of Rehabilitation Research, 36(3), 268-274. https://doi.org/10.1097/mrr.0b013e3283606218

Rajappan, R., Abudaheer, S., Selvaganapathy, K., \& Gokanadason, D. (2015). Effect of mirror therapy on hemiparetic upper extremity in subacute stroke patients. International Journal of Physiotherapy, 2(6), 1041-1046. https://doi.org/10.15621/ijphy/2015/v2i6/80766

Ramachandran, V. S., \& Rogers-Ramachandran, D. (1996). Synaesthesia in phantom limbs induced with mirrors. Proceedings of the Royal Society B: Biological Sciences, 263(1369), 377-386. https://doi.org/10.1098/rspb.1996.0058

Rodrigues, L. C., Farias, N. C., Gomes, R. P., \& Michaelsen, S. M. (2016). Feasibility and effectiveness of adding objectrelated bilateral symmetrical training to mirror therapy in chronic stroke: a randomized controlled pilot study. Physiotherapy Theory and Practice, 32(2), 83-91. https://doi.org/10.3109/09593985.2015.1091872

Salem, A. H. M., \& Huang, X. (2015). The effects of mirror therapy on clinical improvement in hemiplegic lower extremity rehabilitation in subjects with chronic stroke. International Journal of Medical, Health, Biomedical, Bioengineering and Pharmaceutical Engineering, 9(2), 163166. https://doi.org/10.5281/zenodo.1099406

Sciusco, A., Ditrenta, G., Rahinò, A., Damiani, S., Megna, M., Ranieri, M., \& Megna, G. (2008). Mirror therapy in the motor recovery of upper extremities. Europa Medicophysica, 44(3), $1-5$.

Selles, R. W., Michielsen, M. E., Bussmann, J. B. J., Stam, H. J., Hurkmans, H. L., Heijnen, I., de Groot, D., \& Ribbers, G. M. (2014). Effects of a mirror-induced visual illusion on a reaching task in stroke patients: implications for mirror therapy training. Neurorehabilitation and Neural Repair, 28(7), 652-659. https://doi.org/10.1177/1545968314521005

Sütbeyaz, S., Yavuzer, G., Sezer, N., \& Koseoglu, B. F. (2007). Mirror therapy enhances lower-extremity motor recovery and motor functioning after stroke: a randomized controlled trial.
Archives of Physical Medicine and Rehabilitation, 88(5), 555-559. https://doi.org/10.1016/j.apmr.2007.02.034

Thieme, H., Mehrholz, J., Pohl, M., Behrens, J., \& Dohle, C. (2012). Mirror therapy for improving motor function after stroke. Cochrane Database of Systematic Reviews, 14(3), CD008449.

https://doi.org/10.1002/14651858.cd008449.pub2

Thieme, H., Bayn, M., Wurg, M., Zange, C., Pohl, M., \& Behrens, J. (2013). Mirror therapy for patients with severe arm paresis after stroke - a randomized controlled trial. Clinical Rehabilitation, 27(4), 314-324. https://doi.org/10.1177/0269215512455651

Toh, S. F. M., \& Fong, K. N. K. (2012). Systematic Review on the Effectiveness of Mirror Therapy in Training Upper Limb Hemiparesis after Stroke. Hong Kong Journal of Occupational Therapy, 22(2), 84-95. https://doi.org/10.1016/j.hkjot.2012.12.009

Wada, Y., Kondo, I., Sonoda, S., Yamada, K., Narukawa, A., Kawakami, K., Nonoyama, S., Miyasaka, H., Teranishi, T., Nagai, S., \& Takeshima, N. (2011). Mirror therapy for severely affected ankle joints of stroke patients. Japanese Journal of Comprehensive Rehabilitation Science, 2, 71-76. https://doi.org/10.11336/jjcrs.2.71

Yavuzer, G., Selles, R., Sezer, N., Sütbeyaz, S., Bussmann, J. B., Köseoğlu, F., Atay, M. B., \& Stam, H. J. (2008). Mirror therapy improves hand function in subacute stroke: a randomized controlled trial. Archives of Physical Medicine and Rehabilitation, 89(3), 393-398. https://doi.org/10.1016/j.apmr.2007.08.162

Yeldan, I., Huseyinsinoglu, B. E., Akinci, B., Tarakci, E., Baybas, S., \& Ozdincler, A. R. (2015). The effects of very early mirror therapy on functional improvement of the upper extremity in acute stroke patients. Journal of Physical Therapy Science, 27(11), 3519-3524. https://doi.org/10.1589/jpts.27.3519

Yildirim, M., \& Kanan, N. (2016). The effect of mirror therapy on the management of phantom limb pain. A $\check{g} r l: A \breve{g r} l$ (Algoloji) Derneği'nin Yayın organıdır, 28(3), 127-134. https://doi.org/10.5505/agri.2016.48343

Yoo, S. J. (2010). An effect of mirror therapy on upper extremity function and activities of daily living in patients with poststroke hemiplegia [Unpublished master's thesis]. Department of Occupational Therapy, Graduate School of Rehabilitation Science, Daegu University.

Zhang, X., Liang, J., \& Huang, R. (2018a). Effect of acupuncture combined with mirror therapy on functional recovery of posterior lower limb after stroke and observation on nursing effect. Journal of Sichuan Traditional Chinese Medicine, 3, 208-210.

Zhang, R., Liu, B., Chen, W., Zheng, Y., Huang, W., Yu, Y., $\mathrm{Wu}$, L., \& Zhu, M. (2018b). Effects of mirror therapy combined with 'Tong Du Xing Shen' acupuncture therapy on function recovery of upper limbs after stroke. Medical Exploration, 3(1), 16-26. 